\title{
Eugène Boban ou les aventures d'un antiquaire au pays des américanistes
}

\section{Pascal Riviale}

\section{(2) OpenEdition \\ 1 Journals}

Édition électronique

URL : http://journals.openedition.org/jsa/1855

DOI : $10.4000 /$ jsa. 1855

ISSN : 1957-7842

\section{Éditeur}

Société des américanistes

\section{Édition imprimée}

Date de publication : 1 janvier 2001

Pagination : 351-362

ISSN : 0037-9174

\section{Référence électronique}

Pascal Riviale, "Eugène Boban ou les aventures d'un antiquaire au pays des américanistes ", Journal de la société des américanistes [En ligne], 87 | 2001, mis en ligne le 17 novembre 2005, consulté le 01 mai 2019. URL : http://journals.openedition.org/jsa/1855 ; DOI : 10.4000/jsa.1855 


\title{
EUGÈNE BOBAN \\ OU LES AVENTURES D'UN ANTIQUAIRE AU PAYS DES AMÉRICANISTES
}

\author{
Pascal RIVIALE *
}

Si des curiosités américaines ont commencé à circuler en France auprès des amateurs et des collectionneurs dès le $\mathrm{XvI}^{\mathrm{e}}$ siècle, leur intégration dans un circuit marchand n'a débuté qu'au XVIII ${ }^{\mathrm{e}}$ siècle (Riviale 1993). La part des antiquités précolombiennes dans le marché de la curiosité resta toutefois assez minime. Même au $\mathrm{XIX}^{\mathrm{e}}$ siècle, seuls quelques antiquaires sont mentionnés dans les documents pour la possession de telles pièces dans leur fonds de commerce. La première référence que l'on ait concerne un certain Brunswicks, "marchand de curiosités », qui vendit en 1863 au musée Calvet d'Avignon une quinzaine de poteries et de statuettes péruviennes (Riviale 1996). Pour préparer son article sur la céramique du Nouveau Monde, Lucien de Rosny s'était rendu chez plusieurs collectionneurs particuliers et chez quelques marchands, afin d'y observer des pièces intéressantes pour son étude; il mentionnait ainsi M. Alix, antiquaire bien connu sur la place de Paris (Rosny 1875). D'autres commerçants servaient probablement uniquement d'intermédiaires pour mettre en contact des personnes rentrant d'un voyage ou d'un séjour en Amérique latine avec une collection d'antiquités dans leurs valises et des amateurs potentiels ${ }^{1}$.

L'intérêt suscité par les collections ethnographiques au XIX ${ }^{\mathrm{e}}$ siècle a certainement contribué à favoriser le développement de ce secteur particulier du marché de la curiosité, même s'il était encore seulement balbutiant (le nombre des collectionneurs intéressés demeurant assez restreint et les musées disposant de crédits beaucoup trop limités pour faire d'importantes acquisitions). Nous avons montré il y a quelques années, avec le cas des antiquités péruviennes, qu'un nombre assez considérable d'objets circulaient en France depuis le premier tiers du Xıx ${ }^{\mathrm{e}}$ siècle (Riviale 1996) ${ }^{2}$.

Si les marchands s'intéressant aux objets ethnographiques étaient encore rares et difficiles à identifier, aujourd'hui, un individu sort néanmoins de l'ordinaire : par son parcours, l'importance de ses collections et l'ampleur de ses relations au sein de la communauté scientifique, l'antiquaire Eugène Boban constitue sans conteste l'un des personnages les plus étonnants de cette profession, a fortiori lorsque l'on se propose de parler du marché de l'ethnographie et de l'archéologie amérindiennes. En effet, à peine

* Musée d'Orsay, Paris, chercheur associé, EREA (Équipe de recherche en ethnologie amérindienne), UPR 324, CNRS, Villejuif.

Journal de la Société des Américanistes 2001, 87 : p. 351 à 362. Copyright (C) Société des Américanistes. 
avait-il été révélé au monde scientifique que cet antiquaire sut se construire une réputation de spécialiste incontestable dans le domaine de l'ethnographie préhistorique et "exotique » auprès des savants et des conservateurs de musées. De fait, sa correspondance est là pour nous prouver qu'Eugène Boban fut en contact avec les principaux protagonistes de l'américanisme français (et plus largement de la science ethnographique). Enfin, à l'heure où l'on s'efforce de faire le point sur les collections amérindiennes disponibles dans les musées français, il est intéressant de constater qu'un nombre important de musées possède actuellement des objets ayant transité à un moment ou un autre par cet antiquaire ; une étude attentive de l'origine de ces collections - aujourd'hui dispersées - pourrait certainement permettre des recoupements utiles à leur identification. Ce personnage mérite d'autant plus que l'on se penche sur son cas que, parmi les objets mexicains présentés actuellement au pavillon des Sessions du musée du Louvre, plusieurs étaient passés par les mains d'Eugène Boban.

On ne sait rien des premières années mexicaines d'Eugène Boban, de ses activités professionnelles et de son intérêt pour l'archéologie. On doit cependant remarquer que, s'il n'était pas connu des cercles américanistes français, il fit promptement irruption dans la vie de la commission scientifique du Mexique fondée en 1864. Comment était-il entré en contact avec le ministère de l'Instruction publique ? On ne le sait pas précisément. En tout état de cause, les documents d'archives indiquent qu'à la fin de l'année 1865 le ministère lui écrivit pour le remercier de "bien vouloir participer à l'Exposition universelle en envoyant des collections d'antiquités, des fossiles et des dessins ${ }^{3}{ }^{3}$. À partir de ce moment-là, les comptes rendus des séances de la commission furent ponctués par les lettres du colonel Doutrelaine annonçant l'envoi de dessins d'antiquités provenant de la collection Boban, avant que ce ne soient les objets proprement dits qui soient expédiés, grâce aux bons soins des services de transport du corps expéditionnaire français au Mexique (Riviale 1999, p. 329). Un document récapitulatif de ces envois nous permet de comptabiliser pas moins de 2800 pièces ${ }^{4}$. Les accointances de Boban avec le colonel du génie Doutrelaine restent mal définies, on peut toutefois dire que le premier profita dans une certaine mesure des opérations de terrassement effectuées sous la houlette du second : tel paraît avoir été le cas au Cerro de las Palmas et à Santiago Tlatelolco. Ses fonctions annoncées d'« antiquaire de l'empereur Maximilien » pourraient également avoir permis à Eugène Boban de réunir d'importantes collections d'antiquités. Hamy rapporte que, lors de travaux pour la construction d'une route, on avait découvert quelques débris d'antiquités sur le Cerro de Zahuatlan (près de Tlalnepantla); la nouvelle ayant couru que le tumulus contenait un trésor, il fut bientôt rasé avant que les terrassiers envoyés par Maximilien pour y faire des fouilles ne puissent intervenir. Boban put néanmoins recueillir un certain nombre d'objets qui y avaient été trouvés (Hamy 1884, p. 33, note 3), ce qui laisse penser qu'il avait ses entrées auprès des autorités compétentes. Nous disposons à l'heure actuelle de fort peu d'informations sur la réalité des fonctions officielles du personnage, toutefois quelques indices nous permettent de croire à la réalité de son titre ${ }^{5}$. Une annonce publiée par Boban dans un journal (mexicain ?) qualifiait alors ce dernier de « anticuario comisionado por S. M. el Emperador ${ }^{6}$, son magasin étant situé au $n^{\circ} 7$ du callejón del Espíritu Santo, à Mexico. 
Ses collections furent jointes aux objets rapportés par le colonel Doutrelaine, Guillemin-Tarayre et Biart, pour être montrées au public dans le cadre de l'exposition de la commission du Mexique, dans l'enceinte du ministère de l'Instruction publique en 1867 (Riviale 1999, p. 333). Pour Boban, la présentation de ses collections à l'Exposition universelle de Paris représentait un intérêt majeur : c'était pour lui l'occasion de montrer ce type d'antiquités à un très grand nombre de gens et de leur trouver un acheteur. Une lettre adressée à lui par le colonel Doutrelaine ne laisse aucun doute sur cet objectif : «[...] il n'est plus possible d'espérer maintenant que l'acquisition de votre cabinet soit faite ni par le gouvernement, ni par aucun amateur que je sache [...]. Je l'ai [Adrien de Longpérier] fait bondir en lui parlant du prix de $50000 \mathrm{~F}$ auquel vous proposez de céder votre cabinet pour les musées impériaux. Dans de telles conditions, vous n'avez d'autre parti à prendre que d'attendre des jours meilleurs $[\ldots]{ }^{\prime}{ }^{7}$.

Ses collections, récupérées par sa sœur, furent confiées aux bons soins de sa mère avec pour mission de faire son possible pour les vendre. La tâche était pourtant ardue. C'est ce dont l'informait un certain Charles Loysel (apparemment un officier rencontré lors de l'Intervention, devenu par la suite aide de camp du maréchal Forey) qui venait de visiter Mme Boban : «Elle dit qu'elle ne s'y entend pas du tout pour le placement de vos bibelots et que vous devriez dans tous les cas venir pour quelque temps pour vous occuper de vos affaires. Pour moi j'ai le plus grand désir de vous voir réussir comme vous le méritez, aussi je lui répète qu'il faut vous laisser juge de l'opportunité du retour de manière que vous soyez au moins récompensé de tout le mal que vous vous êtes donné... " ${ }^{8}$. L'idée du retour en France faisait son chemin. En attendant qu'elle se concrétise, Boban fit don en 1869 (par l'entremise de sa mère ?) d'une cinquantaine de pièces (provenant majoritairement de Teotihuacan) au musée Saint-Jean, à Angers. Si l'on ne sait pourquoi ce musée plus particulièrement qu'un autre profita de cette donation (sans doute due à une relation sur place), on pourrait tout au moins s'étonner du fait que le musée Américain du Louvre n'ait pas bénéficié d'une telle largesse $^{9}$ : peut-être doit-on attribuer cela au dépit de Boban envers Longpérier qui avait rejeté ses offres de vente. La comparaison des explications jointes aux pièces envoyées au musée d'Angers (Antony 1983) avec le catalogue manuscrit de sa collection rédigé à cette même époque par Boban, dans la perspective d'une vente éventuelle (le catalogue porte l'adresse des deux magasins de Boban : celui ouvert à Paris vers 1870 au 35 de la rue du Sommerard - portant l'enseigne « Antiquités mexicaines » - et son ancien local du callejón del Espíritu Santo, à Mexico), permettrait sans doute d'identifier plus clairement ces objets. Ce catalogue ${ }^{10}$ ne répertorie pas moins de 2025 numéros, il s'agit d'objets en terre cuite, silex, obsidienne, jade, pétrosilex, os (et au moins un objet en cristal de roche ou quartzite). Les provenances annoncées sont extrêmement nombreuses et diverses ; elles correspondent en majorité au Bassin de Mexico, les autres régions représentées sont celles de Veracruz, d'Oaxaca, du Yucatan et, très marginalement, de Durango. En 1871, Boban entra en contact avec un curieux personnage du nom de Jos Karrick Riggs (probablement un collectionneur résidant à Paris), qui s'offrit pour servir d'intermédiaire auprès de diverses institutions américaines afin de vendre les collections proposées par l'antiquaire. En 1872, il lui faisait ainsi part de ses démarches - infructueuses - auprès de l'American Museum of Natural History et du Museum of Arts, à New York, et lui indiquait qu'il restait à 
relancer l'Historical Society : «Cette société est riche et m'a déjà indiqué son désir d'acquérir votre collection " ${ }^{11}$. En 1874, c'était avec le directeur d'un musée new yorkais (non identifié) qu'une entrevue était arrangée à Paris. Finalement la plus grande partie des objets mentionnés dans le catalogue sus-mentionné fut achetée en 1875 par Alphonse Pinart ${ }^{12}$, un jeune homme qui s'était très tôt passionné pour l'ethnographie amérindienne et qui s'était illustré en explorant le Nord-Ouest américain. La collection acquise comprenait une très large majorité de pièces mexicaines ( 1463 numéros, dont le fameux masque de Xipe Totec et la statue de Quetzalcoatl, tout récemment exposés au Pavillon des Sessions du Louvre), puis quelques centaines d'objets d'Amérique centrale et méridionale ${ }^{13}$ (que Boban avait certainement obtenus par le biais de son commerce). Pinart ayant dilapidé sa fortune dans l'achat de la bibliothèque de l'abbé Brasseur de Bourbourg, puis dans celui de la collection archéologique de Boban, ne fut plus en mesure de financer ses propres explorations au Nouveau Monde comme il l'avait initialement prévu ${ }^{14}$. Il se vit donc dans l'obligation de proposer un marché au ministère de l'Instruction publique : il demandait une mission ethnographique dans les deux Amériques pour lui et Léon de Cessac, financée par le ministère, en l'échange du don de ses collections à l'État. Un arrêté en date du 2 avril 1878 chargea donc les deux explorateurs de la mission demandée (Riviale 1996, p. 154). C'est cette collection qui constitua le noyau initial du futur musée d'Ethnographie du Trocadéro (collection portant d'ailleurs symboliquement le numéro d'enregistrement 78-1). Dans le mouvement d'émulation créé par la création de ce musée, plusieurs autres musées français vinrent solliciter le ministère afin de recevoir des " doubles » (ou considérés comme tels) des nombreux objets s'accumulant dans les réserves du musée d'Ethnographie en phase d'ouverture. C'est ainsi qu'en 1881, le musée de la Céramique, à Sèvres, reçut, entre autres, une vingtaine d'objets mexicains (une majorité provenant de Teotihuacan, au moins un de la côte du Golfe, les autres étant d'origine indéterminée) à titre d'échange ${ }^{15}$, tandis que le musée de Lille obtint, à une date inconnue, quelques objets similaires, toutes ces pièces provenant de la collection d'Alphonse Pinart et donc d'Eugène Boban.

Après cette bonne opération commerciale, Boban entreprit ce qu'avec un peu de suspicion, on pourrait peut-être interpréter comme une opération publicitaire auprès des milieux américanistes. En 1875, à l'occasion du premier congrès international des Américanistes, à Nancy, il offrait aux organisateurs du congrès une série de moulages d'objets précolombiens venant de ses collections (moulages qui furent exposés avec les autres objets constituant le "musée Américain de Nancy », lors de l'ouverture du congrès), puis refit de même pour le cinquième congrès (Copenhague, 1883). En 1878, Boban participa à l'exposition spéciale des sciences anthropologiques, dans le cadre de l'Exposition universelle de Paris : y étaient présentées des antiquités mexicaines ainsi que des contrefaçons fabriquées dans les environs de Mexico ${ }^{16}$. Toutes ces pièces étaient également proposées à la vente, puisque on les retrouve dans le catalogue de son Comptoir d'archéologie préhistorique (Boban 1878), publié avant le début de l'exposition.

Il est peu probable que Boban soit retourné au Mexique au cours de ces années-là, il n'avait d'ailleurs pas nécessairement besoin de se rendre sur place pour continuer à alimenter son fonds de commerce, dans la mesure où il procédait à des échanges avec des collectionneurs d'outre-Atlantique ${ }^{17}$. En outre, il se trouvait toujours des voya- 
geurs ou des personnes ayant résidé en Amérique latine pour lui proposer de nouvelles pièces. C'est ainsi qu'il put, vers 1880-1881, faire l'acquisition de la collection d'antiquités précolombiennes réunie par le Dr Fuzier lorsqu'il était en poste à l'hôpital militaire de Veracruz durant l'Intervention française au Mexique. Le Dr Fuzier, décédé en 1880, avait légué ces objets au musée Américain du Louvre, mais ne souhaitant pas accéder à certaines exigences testamentaires, le conservateur avait fini par refuser ce legs (Guimaraes 1994, p. 79). Dès lors, Boban prit certainement contact avec l'héritière de Fuzier pour acquérir cette collection, ce qui fut fait très peu de temps après : la consultation du Catalogue d'ouvrages scientifiques, publié en 1881, par Boban nous permettrait de vérifier si c'était déjà le cas cette année-là, malheureusement nous n'en avons pas encore trouvé d'exemplaire disponible ${ }^{18}$. Une photographie d'une vitrine d'objets mexicains appartenant à Boban prise vers cette période montre clairement certains objets ayant appartenu à Fuzier : on y reconnait notamment les trois statuettes de l'Occident données au Dr Fuzier par le Dr Champenois après une campagne vers Guadalajara durant l'Intervention (Riviale 1999, p. 327), ainsi qu'une grande tête en pierre trouvée à Cosomoloapan ${ }^{19}$.

La renommée de l'antiquaire était maintenant bien assise et de nombreux amateurs et conservateurs de musées s'adressaient à lui pour accroître leurs collections, non seulement des conservateurs des musées français, mais aussi des personnalités étrangères, comme l'anthropologue genevois Gosse, le naturaliste florentin Enrico Giglioli, ou bien l'anatomiste suédois Gustaf Retzius ${ }^{20}$. En 1882, Boban put quitter son magasin de la rue du Sommerard pour un local plus vaste au 85 du boulevard Saint-Michel. Puis vers 1884-1885, pour une raison inconnue, Boban s'installa de nouveau à Mexico où il ouvrit un local oscillant entre le musée et le magasin d'antiquités. Le Museo cientifico ( 10 a de la Violeta, $\mathrm{n}^{\circ} 6$ ") se visitait moyennant un real, mais on pouvait aussi apparemment y acheter des objets. Réparties dans quatre salles, se trouvaient exposées des « curiosités » ethnographiques du monde entier, des antiquités préhistoriques, classiques (grecques, romaines, égyptiennes), précolombiennes, coloniales, des momies et autres pièces anthropologiques et diverses publications scientifiques. Des recherches resteraient à faire au Mexique pour déterminer quelles y furent les activités de Boban durant ces années-là, on peut tout au moins considérer qu'il put établir ou renouer des contacts dans les milieux scientifiques mexicains ${ }^{21}$, en vue de réunir de nouvelles collections. On sait notamment qu'il était en relation avec le directeur du musée national, Gumercindo Mendoza, le responsable des collections d'anthropologie physique du musée, M. Sánchez (à qui il aurait acheté quelques pièces) et avec Leopoldo Batres, qui avait entrepris de vastes fouilles à Teotihuacan (Walsh 1997, pp. 122 et 125). C'est d'ailleurs avec ce dernier qu'il fut mêlé à un scandale de fraude archéologique (en essayant de vendre une contrefaçon de crâne en cristal au musée national de Mexico), scandale qui l'aurait poussé à quitter précipitamment le Mexique en 1886, pour s'installer à New York (Ibid., p. 127). Cette ville fut pour lui le siège de deux grandes ventes aux enchères, au cours desquelles il s'efforça de liquider l'ensemble de ses collections archéologiques et ethnographiques. La première eut lieu du 9 au 11 décembre 1886. Le catalogue de la vente (mentionnant 1360 objets) nous permet de constater que Boban avait emporté avec lui une partie des collections du Dr Fuzier (dont une statuette de l'Occident, des céramiques mayas — probablement fausses - et des antiquités recueillies à Medellín, près de Veracruz), 
ainsi que les urnes zapotèques réunies par M. Martin, un ancien consul de France à Oaxaca. Cette vente n'ayant pas rencontré le succès escompté, Boban reprit contact avec la Smithsonian Institution (en la personne de Holmes) afin de lui céder le restant de ses collections. Les tractations s'étalèrent durant le premier semestre de l'année $1887^{22}$, mais n'aboutirent apparemment pas, ce qui amena Boban à organiser une seconde vente le 11 octobre 1887.

De retour à Paris, Eugène Boban ouvrit rapidement un nouveau magasin si l'on en juge d'après un prospectus ${ }^{23}$ en date du mois de décembre 1887 annonçant l'ouverture du « Comptoir d'archéologie préhistorique et ethnographique » (sis au 122 de l'avenue d'Orléans à Montrouge). Sa rencontre, quelques mois plus tard, avec le collectionneur Eugène Espéridion Goupil allait s'avérer décisive et représenter un tournant dans sa vie professionnelle. Selon un accord établi d'abord oralement le 19 février 1888, Boban lui céda l'ensemble de ses collections mexicaines pour la somme de 8000 francs $^{24}$. Dès lors, tout en poursuivant ses activités commerciales pour son propre compte, le marchand resta pendant dix ans aux côtés de l'amateur; classant ses collections, rédigeant les notices, repérant et négociant pour lui les pièces intéressantes circulant sur le marché. Leur abondante correspondance est là pour attester de ses fonctions d'intermédiaire dans diverses opérations visant à augmenter les collections de Goupil. L'album de ces collections, établi par les soins de Boban, nous permet d'identifier l'origine de la plupart des pièces. On y retrouve un grand nombre d'objets ayant appartenu au Dr Fuzier ${ }^{25}$ (Occident, Veracruz, Yucatan), des urnes zapotèques de la collection Martin ${ }^{26}$, de nombreuses pièces de la vallée de Mexico réunies par Charles Baur (négociant établi à Puebla, avec qui Boban était en affaire), des objets coloniaux rapportés par Génin, etc. (Boban s.d.). Certaines de ces pièces avaient été mises en vente à New York en 1886 et 1887, signe que ces ventes n'avaient pas été d'aussi grandes réussites que ce que clama Boban après coup.

L'antiquaire servit également d'intermédiaire entre Goupil et Aubin pour l'achat de la collection de manuscrits mexicains de ce dernier. Une première rencontre avait eu lieu chez Aubin le 28 mars 1889 et Boban parvint à convaincre Goupil de faire l'acquisition de cette magnifique collection. L'acte de vente fut finalement établi le 11 avril de cette même année et Boban se chargea de classer les documents et d'en dresser le catalogue (publié en 1892).

Dès 1889, Eugène Goupil avait envisagé de léguer ses manuscrits mexicains à la Bibliothèque nationale et ses antiquités précolombiennes au musée d'Ethnographie du Trocadéro ${ }^{27}$. Après le décès de son époux en 1895, Mme Goupil fit effectivement don de l'ensemble des manuscrits à la Bibliothèque nationale en 1898 ; en revanche, le sort réservé à la seconde partie de la collection reste mystérieux : jusqu'à présent nous n'avons pu identifier les pièces archéologiques ayant appartenu à Goupil parmi les objets actuellement présents au musée de l'Homme (mais une recherche plus approfondie s'imposerait pour s'en assurer). Qu'advint-il de cette collection archéologique ? Fut-elle alors vendue comme le fut la bibliothèque américaniste de Goupil (le 4 février 1899) ? Il est possible qu'elle ait été dispersée aux États-Unis. En effet, une information transmise par Jane Maclaren Walsh laisse penser que certaines pièces seraient passées entre les mains de divers collectionneurs américains ${ }^{28}$. Quant à Eugène Boban, il poursuivit ses activités d'antiquaire durant une dizaine d'années encore. En 1908, 
année de son décès, Boban réalisa une ultime vente importante : au début de cette année il vendit au muséum d'Histoire naturelle de Rouen (par l'intermédiaire d'un collectionneur du nom de Loppé) une collection d'antiquités précolombiennes, comptant environ 600 pièces, dont une large majorité de pièces mexicaines et plusieurs dizaines d'antiquités sud-américaines (Antony 1983). La renommée de Boban s'était déjà estompée : à notre connaissance seule une courte notice nécrologique fut publiée par la Revue d'Anthropologie (celui-ci ayant été dans le passé membre de la Société d'Anthropologie de Paris).

Quelle image conserver de cet étrange personnage, dont le parcours et la réussite paraissent si étonnants ? La conclusion prête à discussion. Pour la plupart de ses contemporains, il semble avoir joui d'une grande réputation de connaisseur et de marchand au service de la science, réputation d'ailleurs grandement confortée lorsqu'il publia un ouvrage très érudit sur la collection de manuscrits de M. Goupil (Boban 1892). Ernest-Théodore Hamy, le premier directeur du musée d'Ethnographie du Trocadéro, avait apparemment pleinement confiance en lui (il fit appel à ses lumières pour la préparation de la publication des travaux anthropologiques de la commission scientifique du Mexique en 1884), comme nombre de ses pairs qui avaient si souvent recours à lui pour enrichir leurs collections préhistoriques et ethnographiques. Sa clientèle était en effet composée tant d'amateurs « éclairés » que de professionnels unanimement reconnus et tous - ou presque - avaient en commun de s'en remettre à Boban pour leur procurer de " belles " pièces. Deux demandes extraites de la correspondance reçue par l'antiquaire illustrent clairement notre propos. En 1871, un certain Bruet, fonctionnaire et amateur de lithiques, lui écrivait ces quelques lignes : " J'ai perdu, vous savez, dans l'incendie du ministère des Finances la petite collection que vous m'aviez composée. Et je viens vous prier instamment de m'en composer une nouvelle... » ${ }^{29}$; en 1893, c'est Gabriel de Mortillet qui lui formulait la demande suivante : «Mon cher Boban, Avez-vous encore du préhistorique ? Il me faudrait : un poignard et une pointe de lance en silex du Danemark, [...] des pointes de flèches américaines, lames et nucléus en obsidienne du Mexique [...]. Je n’ai pas besoin de la quantité, mais je voudrais de bons échantillons. C'est pour compléter une série d'études " ${ }^{30}$. Boban semble ainsi avoir occupé une position centrale dans les milieux de l'ethnographie et de l'archéologie préhistorique. Un exemple nous paraît à ce titre très révélateur : ami de longue date de Léon de Rosny, l'antiquaire était membre de la Société d'Ethnographie depuis $1869^{31}$, en même temps qu'il était en étroite relation avec les plus grands anthropologues français (deux tendances scientifiques et intellectuelles qui alors ne se mélangeaient quasiment pas). Une fois de plus, sa correspondance est là pour démontrer la longévité et la fidélité de nombre de ces amitiés nouées dans les milieux scientifiques (ainsi que pendant ses premières années mexicaines: Domenech, Lanusse, Loysel, Becker, Laverrière). Certes, cette correspondance a pu être expurgée en son temps de tout document peu flatteur, mais force est de constater que celle-ci témoigne plutôt d'une grande estime générale en faveur du marchand. Pour certains auteurs actuels, cette image pourrait bien être trompeuse : Boban aurait profité de sa renommée pour écouler des objets frauduleux. Le cas des crânes en cristal ayant transité par le marchand est en effet troublant (Walsh 1997) ; de même, en 1986, E. Pasztory a exprimé ses doutes sur le masque de Xipe Totec (du musée de l'Homme) vendu à Pinart, et a tout récemment souligné le caractère fantaisiste de certaines des 
indications fournies par Boban (Sculptures... 2000, p. 372). Le fait est que l'antiquaire savait assurer sa propre publicité et jouer de ses contacts parmi les américanistes et les préhistoriens pour parvenir à ses fins, le fait est également qu'un certain nombre de contrefaçons ont indéniablement transité par ses magasins successifs. Les avait-il introduites sur le marché en toute connaissance de cause ou s'était-il laissé abuser ? La question reste posée et nous ne saurions pour l'instant trancher. Il n'en reste pas moins que la trajectoire d'Eugène Boban est extrêmement intéressante dans la mesure où ce dernier représente le premier antiquaire du genre qui se soit spécialisé dans les objets " exotiques » et les artefacts préhistoriques, à une époque où les musées abritant ce type de collections étaient en pleine expansion. Il a donc, à sa façon, participé à l'émergence d'un mouvement muséographique qui accordait toute leur valeur aux objets pour eux-mêmes et pour ce qu'ils étaient censés illustrer du développement linéaire de la civilisation, souvent au détriment du contexte de découverte des pièces conservées et exposées. Les erreurs d'appréciation étaient par conséquent fréquentes, a fortiori pour ce qui concerne des cultures amérindiennes dont on connaissait encore peu de chose, ce qui explique que tant de contrefaçons se soient glissées parmi les collections authentiques. C'est dans ce contexte que l'américanisme s'est développé au $\mathrm{XIX}^{\mathrm{e}}$ siècle et l'antiquaire Eugène Boban fait partie intégrante du paysage scientifique de cette période pionnière, quelles qu'aient pu être les pratiques intellectuelles et commerciales utilisées par ce dernier en vue d'assurer sa réussite personnelle.

ANNEXE

Tableau récapitulatif des mouvements de pièces parmi les collections mexicaines réunies par Eugène Boban au cours de sa carrière de marchand et localisation actuelle de ces collections

\begin{tabular}{|c|c|c|}
\hline Mouvements de collection & Origine & Localisation actuelle \\
\hline $\begin{array}{l}\text { 1866-1867: } \\
\text { Envoi à Paris de près de } 2800 \text { pièces par } \\
\text { l'entremise du colonel Doutrelaine }\end{array}$ & $\begin{array}{l}\text { Collection réunie au Mexique } \\
\text { dans les années } 1860 \text {, par- } \\
\text { fois avec l'aide de Doutre- } \\
\text { laine }\end{array}$ & $\begin{array}{l}\text { En grande partie au Musée de } \\
\text { l'Homme (coll. Pinart) }\end{array}$ \\
\hline $\begin{array}{l}\text { vers } 1867 ? \text { : } \\
\text { Don au musée Américain du Louvre de } \\
\text { quelques pièces ( } 4 \text { ou plus?) }\end{array}$ & $\begin{array}{l}\text { Pièces extraites de l'envoi fait } \\
\text { en 1866-67? }\end{array}$ & $\begin{array}{l}\text { Musée de l'Homme } \\
\text { (transfert depuis le Louvre en } \\
1887): \text { coll. } 87138\end{array}$ \\
\hline $\begin{array}{l}1869 \text { : } \\
\text { Don au musée Saint-Jean (Angers) d'envi- } \\
\text { ron } 50 \text { pièces }\end{array}$ & $\begin{array}{l}\text { Collection réunie au Mexique } \\
\text { jusqu'à son installation à Paris } \\
\text { vers } 1870\end{array}$ & Musée Pincé, Angers \\
\hline $\begin{array}{l}1870 \text { : } \\
\text { Vente au Muséum d'Histoire naturelle de } \\
\text { deux masques en plâtre d'indiens otomis }\end{array}$ & $\begin{array}{l}\text { Moulage exécuté durant l'In- } \\
\text { tervention française? }\end{array}$ & $\begin{array}{l}\text { Laboratoire d'Anthropologie } \\
\text { du Muséum national d'His- } \\
\text { toire naturelle (nº } 3654 \text { et } \\
3655)\end{array}$ \\
\hline $\begin{array}{l}\text { 1874: } \\
\text { Don de } 4 \text { objets en obsidienne (du Mexi- } \\
\text { que ?) au musée de l'Artillerie }\end{array}$ & $\begin{array}{l}\text { Pièces recueillies au Mexique } \\
\text { jusqu'à son installation à Paris } \\
\text { vers } 1870\end{array}$ & Musée de l'Armée, Paris? \\
\hline
\end{tabular}




\begin{tabular}{|c|c|c|}
\hline $\begin{array}{l}1875 \text { : } \\
\text { Vente d'environ } 1500 \text { pièces mexicaines à } \\
\text { Alphonse Pinart (plus sans doute les quel- } \\
\text { ques centaines de pièces d'Amérique cen- } \\
\text { trale et du Sud " données » à l'État avec le } \\
\text { reste de sa collection par Pinart en 1878) }\end{array}$ & $\begin{array}{l}\text { Collections réunies au Mexi- } \\
\text { que jusqu'à son installation à } \\
\text { Paris vers } 1870+\text { pièces diver- } \\
\text { ses réunies après son retour à } \\
\text { Paris }\end{array}$ & $\begin{array}{l}\text { Musée de l'Homme (coll. } \\
\text { vendue par Pinart en } 1878 \text { au } \\
\text { ministère de l'Instruction } \\
\text { publique) : coll. } 78-1 \\
\text { Musée de la Céramique, } \\
\text { Sèvres (dépôt du musée d'Eth- } \\
\text { nographie en 1881) : une ving- } \\
\text { taine de pièces } \\
\text { Musée d'Histoire natu- } \\
\text { relle, Lille : quelques pièces }\end{array}$ \\
\hline $\begin{array}{l}1875 \text { : } \\
\text { Don (ou prêt) de divers objets et de mou- } \\
\text { lages d'objets mexicains } \\
\text { pour le } 1^{\text {er }} \text { congrès des Américanistes, à } \\
\text { Nancy }\end{array}$ & $\begin{array}{l}\text { Pièces recueillies au Mexique } \\
\text { jusqu'à son installation à Paris } \\
\text { vers } 1870 \text { et moulages réalisés } \\
\text { en France }\end{array}$ & $\begin{array}{l}\text { Musée historique lorrain, } \\
\text { Palais Ducal, Nancy? }\end{array}$ \\
\hline $\begin{array}{l}1876 \text { : } \\
\text { Vente de } 16 \text { crânes au Muséum d'Histoire } \\
\text { naturelle de Paris }\end{array}$ & $\begin{array}{l}\text { Collection réunie durant l'In- } \\
\text { tervention française }\end{array}$ & $\begin{array}{l}\text { Laboratoire d'anthropologie } \\
\text { du muséum national d'His- } \\
\text { toire naturelle : } n^{\circ} 6428 \text { à } 6443\end{array}$ \\
\hline $\begin{array}{l}\text { Date inconnue : } \\
\text { Don de quelques moulages d'objets en os à } \\
\text { la Société d'Anthropologie de Paris }\end{array}$ & $\begin{array}{l}\text { Moulages réalisés en France ; } \\
\text { pièces présentées à l'exposition } \\
\text { des sciences anthropologiques, } \\
\text { Exposition universelle de } \\
\text { Paris, 1878? }\end{array}$ & $\begin{array}{l}\text { Musée de l'Homme (dépôt de } \\
\text { la Société d'Anthropologie) : } \\
\text { coll } 32.88 \text {. } 5 \text { pièces }\end{array}$ \\
\hline $\begin{array}{l}\text { Date inconnue : } \\
\text { Entrée d'au moins une pièce au musée de } \\
\text { la reine Bérengère, au Mans }\end{array}$ & Origine inconnue & $\begin{array}{l}\text { Musée de la reine Bérengère, } \\
\text { Le Mans }\end{array}$ \\
\hline $\begin{array}{l}1886 \text { et } 1887 \text { : } \\
\text { Ventes publiques à New York }\end{array}$ & $\begin{array}{l}\text { Collections réunies en France } \\
\text { (coll. Martin, Fuzier et autres) } \\
\text { et au Mexique (en 1885?) }\end{array}$ & $\begin{array}{l}\text { Apparemment peu de pièces } \\
\text { vendues }\end{array}$ \\
\hline $\begin{array}{l}1888 \text { : } \\
\text { Vente d'une très importante collection à } \\
\text { Eugène Goupil }\end{array}$ & $\begin{array}{l}\text { Collections réunies en France } \\
\text { (coll. Martin, Fuzier et autres) } \\
\text { et au Mexique (en 1885?) }\end{array}$ & $\begin{array}{l}\text { Collection apparemment dis- } \\
\text { persée : certaines pièces se- } \\
\text { raient signalées au États-Unis. }\end{array}$ \\
\hline $\begin{array}{l}\text { 1908: } \\
\text { Vente d'environ } 500 \text { pièces à la ville de } \\
\text { Rouen (plus une centaine de pièces du } \\
\text { reste de l'Amérique) }\end{array}$ & Origine inconnue & $\begin{array}{l}\text { Musée d'Histoire naturelle, } \\
\text { Rouen }\end{array}$ \\
\hline
\end{tabular}

NB : ne sont évidemment pas mentionnées ici les ventes à des particuliers réalisées dans le cadre des activités de son magasin)

\section{NOTES}

1. Dans les années 1830 , Edmé Jomard se fit l'écho auprès des autorités compétentes de la présence à Paris de quelques collections mexicaines et centre-américaines (celles de Latourd-Allard et de Franck) proposées à la vente ; 25 ans plus tard c'est la collection Pingret (peintre ayant vécu au Mexique) qui s'attira une certaine publicité auprès des connaisseurs. Bien d'autres collections sont ainsi mentionnées allusivement dans les documents du $\mathrm{XIX}^{e}$ siècle, sans que l'on sache très clairement par quelles mains passaient ces antiquités pour leur mise en vente.

2. Ce qui explique sans doute pourquoi, lorsque l'on fait l'inventaire des collections amérindiennes présentes aujourd'hui dans les musées français, on rencontre tant de donateurs ayant réuni leurs collections par voie indirecte.

3. Lettre du 13 novembre 1865. Archives nationales, Paris, F 172910.

4. «Inventaire des pièces de la collection Boban qui ont été envoyées à la commission scientifique du Mexique " (musée de l'Homme, département Amérique, dossier technique 78.1). 
5. Cette information est notamment donnée par Albert Réville dans un article publié par le journal Le Temps (13 avril 1898). Une carte de visite de Boban, conservée à la Bibliothèque nationale de France, porte la mention " anticuario de S. M. el Emperador 》 (Département des Manuscrits Occidentaux DMO, n.a.f 21 476, pièce $n^{\circ} 231$ ) ; en outre, Auguste Génin fait référence à l'Anuario imperial de Mexico pour 1865 dans lequel Eugène Boban apparaît sous cette même qualité (A. Génin, Les Français au Mexique du xvf siècle à nos jours, Paris, Nouvelles éditions Argo, 1933, p. 344).

6. Coupure de journal (11 mars 1866) insérée dans un des volumes des papiers d'Eugène Boban. Bibliothèque nationale de France, DMO, n.a.f 21476 , pièce $n^{\circ} 255$.

7. Lettre de Doutrelaine (Paris, 14 octobre 1867). Ibid., n.a.f. $21477, \mathrm{n}^{\circ} 101$.

8. Lettre de Charles Loysel (Paris, 14 avril 1869). Ibid., n.a.f. $21478, \mathrm{n}^{\circ} 204$.

9. Seuls quatre objets furent remis - à une date inconnue - par Boban au musée du Louvre. Ils sont actuellement enregistrés au musée de l'Homme, sous le $n^{\circ} 87-138$.

10. "Archéologie mexicaine. Collection E. Boban Duvergé. Catalogue». Ce manuscrit, d'abord conservé à la bibliothèque du musée de l'Homme, est maintenant au département Amérique du musée.

11. Lettre de Jos Karrick Riggs à Boban (Paris, mars 1872). Bibliothèque nationale de France, DMO, n.a.f. $21479\left(n^{\circ} 101-102\right)$.

12. «Vous pouvez, d'après la présente, considérer l'affaire dont nous nous sommes entretenus hier comme conclue aux conditions acceptées de part et d'autre ", lettre d'Alphonse Pinart à Boban (Hôtel du chemin de fer du Nord, 21 avril 1875). DMO, n.a.f. 21478 ( $\left.{ }^{\circ} 556\right)$.

13. 46 numéros pour le Guatemala, 113 pour l'ensemble Équateur-Colombie-Venezuela, 76 pour le Pérou, 75 pour le Brésil, etc., selon un inventaire fait par E. T. Hamy (lettre du 26 juin 1885). Archives nationales, Paris. F 172997 . Il n'est certes pas formellement établi que tous ces objets lui avaient été vendus par Boban, mais cela est très plausible pour la majorité d'entre eux : en effet, compte tenu de sa réputation, le marchand se voyait facilement proposer des pièces archéologiques et ethnographiques provenant de toutes les régions du Nouveau Monde. Pour les objets de la collection Pinart ayant transité par Boban, voir le catalogue Sculptures..., pp. 372-374, 388-389, 405-409, 412-414.

14. En fait, Pinart eut également les plus grandes diflicultés à solder sa dette envers Boban, une lettre datée de 1878 indique que l'explorateur n'avait toujours pas fini de payer sa collection : «... vous pouvez compter sur ce que je vous ai déjà dit que je ferai tout mon possible pour régler entièrement avec vous avant la fin de l'année. " (lettre d'A. Pinart, 22 mai 1878) ; le marchand semble avoir fini par entreprendre une action contre Pinart (lettre de ce dernier, San Francisco, 8 juin 1879). DMO, n.a.f. 21478 ( $n^{\circ} 564$ et 559 ).

15. Arrêté du 14 novembre 1881. Archives nationales, Paris. F 173 846-1. Le fragment d'objet de la côte du Golfe proviendrait du Cerro de los Idolos, à Extanzuelas (Veracruz); il est à noter que d'autres pièces trouvées sur ce même site par José María Melgar se retrouveront dans la collection achetée par Eugène Goupil à Boban en 1888 .

16. Exposition universelle de 1878. Catalogue spécial de l'exposition des sciences anthropologiques, Paris, Imp. nationale, 1878, p. 27.

17. Tel ce M. E. A. Barber, de Philadelphie. Voir Correspondance avec Boban, Bibliothèque nationale de France, DMO, n.a.f. $21476\left(\mathrm{n}^{\circ} 88-92\right)$.

18. Ce catalogue est cité par J. M. Walsh (1997, p. 124) : il paraît d'autant plus intéressant que l'on y mentionnerait un autre crâne de cristal que celui vendu à Alphonse Pinart quelques années auparavant.

19. Papiers Boban. Bibliothèque nationale de France, DMO, n.a.f. 21476 (pièce $n^{\circ} 266$ ). Les objets mentionnés en exemple se retrouvent dans l'album de dessins réalisé par Fuzier (« Mexique. Collection de dessins d'après nature faits ou recueillis de 1862 à 1867 par le Dr Fuzier [...] », bibliothèque du musée de l'Homme, ms 181), fig. 1 à 4 et 302. La photographie de la collection Boban évoquée ici a été publiée dans le catalogue Sculptures... p. 26 ; les statuettes de l'Occident se trouvent dans la partie médiane de la vitrine, à gauche.

20. Lettres à Boban. Bibliothèque nationale de France, DMO, n.a.f. 21477 (pièces nº 27 et 298) et 21479 $\left(\mathrm{n}^{\circ} 70\right.$ et 77$)$.

21. En 1876, il avait été nommé membre honoraire de la Sociedad mexicana de geografia y estadística (lettre d'Ignacio Manuel Altamirano, Mexico, 16 février 1876. Bibliothèque nationale de France, DMO, n.a.f. $\left.21476, \mathrm{n}^{\circ} 50\right)$.

22. Lettres de Holmes à Boban (mars à juillet 1887). Bibliothèque nationale de France, DMO, 21477.

23. Bibliothèque nationale de France, DMO, n.a.f. $21476, \mathrm{n}^{\circ} 165$.

24. Bibliothèque nationale de France, DMO, n.a.f. 21 480, folio 16.

25. Pas tous cependant : certaines pièces pourraient avoir été vendues à New York en 1886 (comme par exemple une des trois statuettes de l'Occident, qui n'apparaît pas dans l'album de la collection Goupil), 
d'autres (ou au moins une : une tête en terre cuite du Veracruz) seraient entrées dans la collection du comte de Lestrange (Exposition universelle de 1889... Section I : anthropologie, ethnographie, p. 129).

26. Ce sont ces mêmes urnes que Max Uhle aurait bien aimé acheter pour le compte du Volkerkunde Museum de Berlin. En 1888, à l'occasion du congrès international des américanistes se tenant cette année-là à Berlin, Boban avait fait circuler des photographies de ses collections. Uhle, intéressé, écrivit à l'antiquaire pour lui demander le prix de certaines de ses pièces (en particulier les pièces n ${ }^{\circ} 108,110,113$ et 116 de son catalogue de vente de 1886); il ne savait pas encore que l'ensemble de la collection venait d'être acheté par M. Goupil. Quelques jours plus tard, ayant appris la nouvelle, Uhle écrivit de nouveau à Boban : « Je regrette que ces belles pièces zapotèques ont pris la place dans une collection, qui paraît être importante, mais n'est pas publique. Je souhaite la publicité à tous vos beaux objets mexicains ". Lettres des 17 [octobre ?] et 5 [novembre ?] 1888. Bibliothèque nationale de France, DMO, n.a.f. 21479 (n 371 et 374 ).

27. Communication personnelle de Monique Cohen (conservateur général, responsable de la division orientale du département des Manuscrits à la Bibliothèque nationale de France).

28. Dans un courrier, Mme Walsh (19 mai 1999) m'avait signalé que l'exemplaire annoté par William Henry Holmes du catalogue de la vente Boban à New York en 1886 portait en regard de certains objets le nom d'un acheteur (un certain M. Barton, un M. Madison, etc.) ; or les objets en question n'ont pu être acquis lors de cette vente, puisque Boban était rentré en France avec et les avait vendus à Goupil en 1888. Reste donc l'hypothèse d'une vente ultérieure, qu'il conviendrait de vérifier. Je saisis ici l'occasion de remercier Jane M. Walsh pour ces précieuses informations.

29. Lettre de M. Bruet (Médéan, 24 juin 1871). Bibliothèque nationale de France, DMO, n.a.f. 21476 $\left(n^{\circ} 414\right)$.

30. Lettre de G. de Mortillet (Saint-Germain-en-Laye, 7 juin 1893). Ibid., n.a.f. 21478 ( ${ }^{\circ} 372$ ).

31. La date de 1863 donnée a posteriori dans l'Annuaire de la Société d'Ethnographie pour 1873 paraît un peu douteuse ; il pourrait en être de même pour la date d'entrée à la Société Américaine de France.

\section{BIBLIOGRAPHIE}

Antony, A., 1983. - Inventaire et étude des collections américaines du muséum d'Histoire naturelle de Rouen, mémoire de maîtrise, Centre de recherches en archéologie précolombienne, université de Paris I.

Besson, C. \& P. Godard,1980. - Le musée Pincé, Angers ; le musée de Tessée ; le musée de la Reine Bérengère, Le Mans; le musée des Beaux-Arts de Rennes, mémoire de maîtrise, Centre de recherches en archéologie précolombienne, université de Paris I.

Boban, E., 1878. - Comptoir d'archéologie préhistorique, Paris, Imp. de Mme veuve BouchardHuzard.

—, 1885. - Museo cientifico..., Mexico, Tip. de A. Vanegas.

-, 1886. - The Boban collection of antiquities, curios and coins, also books, manuscripts and printed, New York, George A. Leavitt \& Co.

—, 1892. - Documents pour servir à l'histoire du Mexique. Catalogue raisonné de la collection de M. Eugène Goupil (ancienne collection de J. M. A. Aubin... ), Paris, Ernest Leroux. 2 vol., un atlas et un carton.

-, s.d. - Collection E. Eugène Goupil à Paris. Statues, statuettes et figurines mythologiques en roches dures, terres cuites et métaux. Armes, objets de parure, instruments de musique et ustensiles divers. Ancienne collection Boban, s.l.

Exposition universelle de 1889 à Paris. Catalogue général officiel. Exposition rétrospective du travail et des sciences anthropologiques. Section I: anthropologie, ethnographie, Lilie [Lille ?], imp. L. Danel. 
Guimaraes, S., 1994. - Le musée des antiquités américaines du Louvre (1850-1887). Une vision du collectionnisme américain $a u x I X^{e}$ siècle, mémoire de maîtrise, Centre de recherches en archéologie précolombienne, université de Paris I.

HaMy, E. T., 1884. - Mission scientifique du Mexique et dans l'Amérique centrale. Recherches zoologiques. $1^{r e}$ partie : anthropologie du Mexique, Paris, Imp. nationale.

Martinoli, V., 1991. - Inventaire des céramiques américaines du musée national de Céramique à Sèvres, mémoire de maîtrise, Centre de recherches en archéologie précolombienne, université de Paris I, 2 vol.

Pasztory, E., 1982. - «Three aztec masks of the god Xipe » in : E. Boone (ed.), Falsifications and misreconstructions of pre-columbian art, pp. 77-105, Washington, Dumbarton Oaks, Trustees for Harvard University.

RÉville, A., 1898. - «Antiquités mexicaines. Les aventures d'une collection », Le Temps, 13 avril 1898.

Reyniers, F., 1966. - Sèvres. Céramiques américaines. Musée national de Céramique. Paris, éd. des musées nationaux (Inventaire des collections publiques françaises $n^{\circ} 12$ ).

Riviale, P., 1993. - «Les antiquités péruviennes et la curiosité américaine sous l'Ancien Régime ", Histoire de l'Art, 21/22, pp. 37-45.

—, 1996. - Un siècle d'archéologie française au Pérou (1821-1914). Paris, L’Harmattan.

—, 1999. — « La science en marche au pas cadencé : les recherches archéologiques et anthropologiques durant l'Intervention française au Mexique (1862-1867) ", Journal de la Société des Américanistes, 85, pp. 307-341.

RoSNY, L. DE, 1875. - « Introduction à l'histoire de la céramique chez les indiens du Nouveau Monde ", Archives de la Société Américaine de France, 2e série, I, pp. 147-185.

Sculptures. Afrique, Asie, Océanie, Amériques. Catalogue de l'exposition au Pavillon des Sessions du Louvre (sous la direction de Jacques Kerchache), 2000, Réunion des musées nationaux, musée du Quai Branly, Paris.

WALSH, M. J., 1997. - «Crystal skulls and other problems» in : A. Henderson \& A. L. Kaepler (ed.), Exhibiting dilemmas. Issues of representations at the Simithsonian Institution, pp. 116-139, Washington \& London, Smithsonian Institution Press. 